\title{
Hit Me Baby One More Time: A Haptic Rating Interface
}

\author{
Christoph Bartneck ${ }^{1}$, Philomena Athanasiadou ${ }^{1}$, and Takayuki Kanda ${ }^{2}$ \\ ${ }^{1}$ Department of Industrial Design \\ Eindhoven University of Technology \\ Den Dolech 2, 5600 MB Eindhoven, The Netherlands \\ christophabartneck.de, p.athanasiadou@student.tue.nl \\ ${ }^{2}$ ATR Intelligent Robotics and Communications Labs \\ 2-2-2 Hikaridai Seikacho Sorakugun, Kyoto, Japan \\ kanda@atr.jp
}

\begin{abstract}
As the importance of recommender systems increases, in combination with the explosion in data available over the internet and in our own digital libraries, we suggest an alternative method of providing explicit user feedback. We create a tangible interface, which will not only facilitate multitasking but provide an enjoyable way of completing an otherwise frustrating and perhaps tiresome task.
\end{abstract}

Keywords: explicit feedback, recommender system, tangible interface.

\section{Introduction}

Recommender systems play a key role in academia and industry. One of the best known recommender sytems is the, "Customers who bought this item also bought..." application from Amazon. The research area of Ambient Intelligence is based on user adaptation and anticipation [1]. The success of the algorithms responsible for the recommendations and adaptations depend on information they gather from the user. They may either do this implicitly through observation, such as logging users' buying behavior, or explicitly by recording direct ratings from the users. Explicit feedback can be very laborious for the users [2,3] and hence implicit feedback is often preferred. However, not all desirable knowledge about the users' state manifests itself in observable behavior. The decision of which music a certain person likes in a specific situation may depend on factors that are not detectable through current sensor technology. A certain lighting condition may, for example, evoke a particular sentimental memory, which causes the user to pick a piece of music from that period. Explicit feedback may help to overcome limitations of implicit feedback-based recommendation systems.

One way to overcome problems associated with gathering data from users is to turn the task into a fun activity, as was presented convincingly by Ahn [4-6]. We developed a haptic interface to turn the process of giving feedback into a fun activity (see Figure 1). We took inspiration from a game often found in Sega World arcades called, "Hammer the Gopher". In this game, the player has to hit gophers with a 


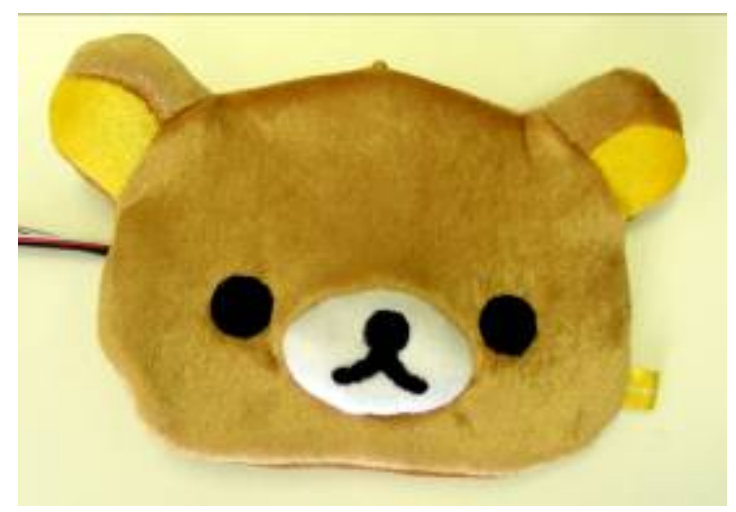

Fig. 1. The Haptic Interface

hammer as soon as they emerge from their holes. Hitting a cute character is a fun activity and the strength of the hit can easily be mapped to a negative rating. A second inspiration came from Nintendo's "Dogs", and "Aibo". In both cases the user can encourage a certain behavior of the creature by petting it. The number of strokes can then easily be mapped onto a positive rating. Our haptic interface is embedded into a flat teddy bear and incorporates a touch sensor and a force sensor. The touch sensor is located in the forehead and responds to even the slightest strokes. The force sensor is embedded into the teddy's nose, since the nose is the teddy's highest point. Through a series of test we were able to develop an algorithm that reliably distinguished between strokes and hits based on the sensor readings.

\section{Need for Effortless, Fast Rating}

Over the past few years we have observed an explosion in the amount of data and media available over the internet. Not only have online resources increased but our digital libraries at home have also grown exponentially. The tiny iPod Nano, with its slim design allows for storage of up to $8 \mathrm{~GB}$, which translates to approximately 2000 songs. With the regular iPod providing up to $80 \mathrm{~GB}$ of storage, which translates to 20.000 songs or 100 hours of movies, we can assume that $8 \mathrm{~GB}$ is only a fraction of what people have stored on their PCs. It is evident that not only "mobile" media players (iPods etc.) but also the media we have safely secured on our PCs has increased dramatically. A quick review of several forums $[7,8]$ that discuss the number of songs in the members' music libraries reveals that on an average people have about 5400 songs. To navigate through this bulk of information, recommender systems can prove invaluable. As the thought of rating 5400 songs in one go could seem overwhelming or even be branded, "mission impossible", it seems essential to have a fast, simple, effortless way of rating music. To that end, the development of an interface that permits the users to rate the music while listening to it, without distracting them from their daily PC activities, sounds ideal. 


\section{Tangible Interfaces Facilitate Multitasking}

By using a simple tangible interface for rating music, the task of rating becomes simple and fast without taking the users' attention away from other activities. Imagine the following scenario: Someone is busy searching for information on the internet and reading documents while listening to music. The interface we created is located next to his PC. While listening to some music, the user realizes he really likes it and wishes to provide/record a rating. There is no need for the user to change application and go through a set of menus in order to rate the music; all he needs to do is simply reach out and pet the teddy bear without taking his eyes off the screen, thus making the process seamless. The existence of peripheral devices can increase the user's ability to multitask as indicated by the creation of Kimura, on office environment that uses peripheral displays in order to facilitate common multitasking practices. The success of Kimura and its advantages compared to other similar projects like Rooms [9], lies in the fact that it does not rely on displaying even more information in the already overloaded desktop, but instead distributing it in the environment [10]. In the same way, by distributing activity to the environment via a peripheral tangible interface, multitasking is facilitated. "Moreover, constraining the interaction to the desktop is a poor match for common human behaviors such as using large amounts of physical space to simultaneously organize, monitor and manage multiple activities" $[10,11]$.

\section{Need for Explicit Feedback}

The need for explicit feedback has been made evident through extensive research in the field of recommender systems. Quiroga and Mostafa [12], compared 3 different methods for user profiling using a) only explicit feedback, b) only implicit feedback and c) a combination of the two. The results showed that the method using explicit feedback initially proved superior but at a certain stage reached a plateau, which surpassed only by the combined method. Combining implicit with explicit feedback is also supported by Zigoris and Zhang [13], who point out the necessity of explicit feedback at the early stages where recommender systems are still getting to know the user. Therefore it makes sense to concentrate efforts on creating an interface that will provide an easy, fast and pleasurable way of giving feedback. By creating this interface, we have attempted to satisfy/fulfill this need. By highlighting the possibility and benefits of using a tangible interface, we hope to inspire future research in this direction.

\section{Evaluation}

In a pilot study, we asked the participants to rate music using either our haptic interface (See Figure 1) or a standard graphical user interface (GUI). The experiment was set up with the type of interface as the only within participant condition. The participants were allowed to rate as many or as few songs as they wished. The following measurements were automatically logged by the software: the number of 
songs rated, the total duration the participant spends on rating the songs, the average duration the participant spends on rating each song, the number of positive and negative ratings, and the total intensity of positive ratings. It did not make sense to compare the intensities of negative ratings, since the GUI provided very different data (scale) compared to the haptic interface (force sensor data). Two female and sixteen male participants, between the ages 21 and 42 years (mean 27.1), partook in the experiment. They were all associated with ATR, Japan. An analysis of variance (ANOVA) revealed that there were not significant differences between the measurements across the two conditions. Table 1 shows the mean and standard errors of the mean (SEMs) for all measurements in both conditions.

Table 1. Means and standard errors of all measurements in both conditions

\begin{tabular}{lrrrr}
\hline Measurement & Haptic & Std. error & GUI & Std. error \\
\hline Number of Songs & 43.61 & 10.04 & 45.83 & 13.92 \\
Number of Positive Ratings & 22.06 & 3.77 & 22.06 & 3.67 \\
Intensity of Positive Ratings & 34.00 & 5.01 & 50.61 & 7.95 \\
Total Duration of Ratings (sec) & 982233.50 & 112273.46 & 997538.33 & 117572.21 \\
Average Duration per Song (sec) & 28803.60 & 3077.73 & 33407.38 & 4467.52 \\
Number of Negative Ratings & 21.56 & 6.78 & 23.78 & 11.08 \\
\hline
\end{tabular}

\section{Conclusion}

The participants were given the specific task of rating music, which naturally favors efficient rather than enjoyable interaction. Even with such a focused task, the haptic interface did perform as well as the GUI. It can be expected that the haptic interface may perform better than the GUI if it is integrated into the daily activities of the users. Next, we intend to perform a long-term study, in which the haptic interface will be available to the users during their daily computer-based work.

As other studies also suggest $[14,15]$, there seems to be a preference towards tangible interfaces when compared to GUIs. The haptic interfaces appear to score higher on 'hedonic quality' [16]. Furthermore, by integrating this interface into the users' daily activities we will be able to study whether this interface facilitates multitasking as expected. The interface is of course not limited to rating music, but can be used for any kind of explicit feedback.

By answering the call for explicit feedback with the creation of a fun and easy-touse tangible interface that is expected to support multitasking, we have opened a new way for the improvement of recommender systems. Most research in recommender systems, as discussed in the introduction, merely refers to recommender system types and algorithms. This paper indicates the potential benefits in using a tangible interface for supporting recommender systems and thus points out the importance of studying and exploring not only the recommender types and algorithms, but also the interface, in order to improve recommender systems. 
Acknowledgements. This research was in part supported by the Japan Society for the Promotion of Science, Grants-in-Aid for Scientific Research No. 18500024. We would like to thank Aoife Currid for proofreading.

\section{References}

[1] Aarts, E., Harwig, R., Schuurmans, M.: Ambient Intelligence. In: Denning, P. (ed.) The Invisible Future, pp. 235-250. McGraw Hill, New York (2001)

[2] Adomavicius, G., Tuzhilin, A.: Toward the next generation of recommender systems: a survey of the state-of-the-art and possible extensions. Knowledge and Data. Engineering, IEEE Transactions on 17, 734-749 (2005)

[3] Nichols, D.M.: Implicit Rating and Filtering.In: presented at 5th Delos Workshop on Filtering and Collaborative Filtering, Budapest, Hungary (1997)

[4] Ahn, L. v., Liu, R., Blum, M.: Peekaboom: a game for locating objects in images. In: Presented at Proceedings of the SIGCHI conference on Human Factors in computing systems, Montreal, Quebec, Canada (2006)

[5] Ahn,L.v., Kedia, M., Blum, M.: Verbosity: a game for collecting common-sense facts. In: presented at Proceedings of the SIGCHI conference on Human Factors in computing systems, Montreal, Quebec, Canada (2006)

[6] Ahn, L.v., Ginosar, S., Kedia, M., Liu, R., Blum, M.: Improving accessibility of the web with a computer game. In: presented at Proceedings of the SIGCHI conference on Human Factors in computing systems, Montreal, Quebec, Canada (2006)

[7] Mac OS X Hints, How much music do you have? (2005) Retrieved February 2007, from http://forums.macosxhints.com/archive/index.php/t-44861.html

[8] My ADSL, How much music do you have? (2006) Retrieved February 2007, from http://mybroadband.co.za/vb/showthread.php?t=63616

[9] Henderson, D.A., Card, S.: Rooms: the use of multiple virtual workspaces to reduce space contention in a window-based graphical user interface. ACM Transactions on Graphics 5, 211-243 (1986)

[10] MacIntyre, B., Mynatt, E.D., Voida, S., Hansen, K.M., Tullio, J., Corso, G.M.: Support for multitasking and background awareness using interactive peripheral displays. In: 14th annual ACM symposium on User interface software and technology, Orlando, Florida, ACM Press, New York (2001)

[11] Mynatt, E.D.: Writing on the Wall, presented at Interact, Edinburgh (1999)

[12] Quiroga, L.M., Mostafa, J.: Empirical evaluation of explicit versus implicit acquisition of user profiles in information filtering systems. In: Proceedings of the fourth ACM conference on Digital libraries, Berkeley, California, United States, ACM Press, New York (1999)

[13] Zigoris, P., Zhang, Y.: Bayesian adaptive user profiling with explicit \& implicit feedback. In: Proceedings of the 15th ACM international conference on Information and knowledge management, Arlington, Virginia, USA, ACM Press, New York (2006)

[14] Jacob, R.J.K., Ishii, H., Pangaro, G., Patten, J.: A tangible interface for organizing information using a grid. In: Proceedings of the SIGCHI conference on Human factors in computing systems: Changing our world, changing ourselves, Minneapolis, Minnesota, USA, ACM Press, New York (2002)

[15] Waldner, M., Hauber, J., Zauner, J., Haller, M., Billinghurst, M.: Tangible Tiles: Design and Evaluation of a Tangible User Interface in a Collaborative Tabletop Setup,presented at OzChi2006, Sydney (2006)

[16] Vastenburg, M.H., Ross, P.R., Keyson, D.V.: A user experience-based approach to home atmosphere control, Universal Access in the Information Society (2007) 